\title{
Obtaining the Fluorescent Chitosan for Investigations in the Analytical Ultracentrifuge
}

\author{
Murodkhon R. Kodirkhonov \\ Namangan State University, Namangan, Uzbekistan \\ Email: kodirkhonov@mail.ru
}

How to cite this paper: Kodirkhonov, M.R. (2019) Obtaining the Fluorescent Chitosan for Investigations in the Analytical Ultracentrifuge. Advances in Biological Chemistry, 9, 23-30. https://doi.org/10.4236/abc.2019.91002

Received: December 21, 2018

Accepted: January 13, 2019

Published: January 16, 2019

Copyright ( 2019 by author(s) and Scientific Research Publishing Inc. This work is licensed under the Creative Commons Attribution International License (CC BY 4.0).

http://creativecommons.org/licenses/by/4.0/

\section{cc) (i) Open Access}

\begin{abstract}
1) In order to achieve the visibility of the chitosan macromolecule for the UV optical system of the analytical ultracentrifuge on investigation of the molecular characteristics and polymers interactions, the labeling of chitosan by a new fluorophore of fluorescein-5-isothiocyanat was carried out. 2) Samples of fluorescent chitosan with two different degrees of fluorophore substitution and various degrees of acetylation were obtained. 3) The labeled chitosans with the fluorescein-5-isothiocyanat allowed estimating the sedimentation coefficient and the molecular characteristic in the analytical ultracentrifuge. 4) The sensitivity of the UV-optical system of the analytical ultracentrifuge for the obtained fluorescent samples of chitosan relatively to the fixation of the meniscus and the influence of the wavelength and rotation speed were estimated.
\end{abstract}

\section{Keywords}

Polysaccharide, Chitosan, Analytical Ultracentrifuge,

Fluorescein-5-Isothiocyanat, Fluorescent Chitosan, Labeling, Sedimentation, Degree of Substitution, UV Absorption

\section{Introduction}

Due to the features of the chemical structure and organization of the macromo lecules, chitosan possesses many properties, that enable to use in many spheres [1]-[6]. Chitosan is polysaccharide consisting of 2 -acetamido-2-deoxy- $\beta$-D-glucopyranose and 2 -amino-2-deoxy- $\beta$-D-glucopyranose units obtained with partial deacetylation of chitin. Such kind of chemical composition conditions some problem in completely quantitative analysis and identification of chitosan. That's why the chemical structure of chitosan lacks the fluorophore group with necessary UV extinction, and as a result the molecule becomes transparent to the 
UV spectrum. Such invisibility of the chitosan also limits investigations of molecular characteristics and other advantaged [7] [8] applications of analytical ultracentrifuge using of UV optical system.

As is known, one possible way of overcoming this invisibility is to label the polysaccharide with a chromophore containing compounds. At present time a labeling procedure effectively used in the field of polymers and on the strength of practical needs this method which develops with searching of the new systems of fluoroagents and polymers.

For example, a non-invasive analytical tool was developed to assess the use of in situ biomaterials for surgical implants or scaffolds in tissue engineering and on the basis of polymeric methods of treatment. In this study, a method for fluorescence monitoring of the chitosan membrane framework degradation was established for in vitro use in bioreactors and, ultimately, in vivo. The basis of this tracking system is the fluorescence-radiating biomaterial obtained by covalent binding of tetramethylboramine isothiocyanate (TRITC) fluorophore based on chitosan [9]. In the work of Coelfen et al. (1996), incorporation of the fluorophore 9-anthraldehyde onto chitosans has been considered and the effect of increase in degree of substitution of the fluorophore has been investigated on 2 chitosans of differing degrees of acetylation by analytical ultracentrifugation. Four chitosans with chemical composition ranging from a fraction of $\mathrm{N}$-acetylated units (FA) of 0.01 to 0.61 were used to prepare fluorescence labeled chitosans with 9-anthraldehyde. The efficiency of the labeling of chitosans was determined by UV and ${ }^{1} \mathrm{H}$ NMR spectroscopy and the influence of substituted fluorescent amounts to conformational characteristic of labeled chitosans in solution was investigated [10].

At present work the samples with high extinction of UV-adsorption fluorescent chitosan modified with fluorescein-5-isothiocyanat (FITC) were obtained and possibilities of using this fluorescence labeled chitosans for characterization in the analytical ultracentrifuge were investigated. Labeled with FITC chitosans intended to use especially for identification of polymers interactions by synthetic boundary methods in AUS [8].

\section{Experimental}

\subsection{Materials}

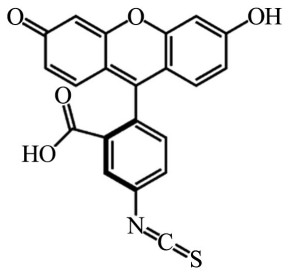

Fluorescein-5-isothiocyanat. As fluoroagent is used fluorescein-5-isothiocyanat (FITC, the product of ALDRICH, F22502-1G), M = 389.4 g/mol.

Chitosan samples with different viscosities $(\eta)$ and fractions of $\mathrm{N}$-acetylated 
units $\left(\mathrm{F}_{\mathrm{A}}\right)$, were provided by SIGMA. The chitosan- $1, \eta=400 \mathrm{mPa}, \mathrm{F}_{\mathrm{A}}=0.15$; The chitosan-2, $\eta=200 \mathrm{mPa}, \mathrm{F}_{\mathrm{A}}=0.18$.

Chitosans were labelled with the fluorescein-5-isothiocyanat by method described in work (Coelfen [10]) and efficiency of labelling process was observed with UV spectroscopy and analytical ultracentrifuge by the technique described in [7] [8]. This was done for each chitosan for two degrees of substitution: $-0.5 \%$ and $-1 \%$, with the amount of label incorporated assayed by its absorption at a wavelength of $240 \mathrm{~nm}$, where the labelled chitosan has a strong absorption maximum.

So, chitosan is hygroscopic polymer therefore for correlation density and solutions concentration the thermogravimetric analysis has performed the dates which are given in Table 1 . The values of density and partial specific volume of the used chitosans samples also are presented in this table. Thermo-Mikrowaage TG 209 F1 (NETZSCH, Germany) and Densitymeter DMA 5000 (Anton Paar) equipments were used for the thermogravimetric and density analysis correspondingly.

\subsection{Solutions}

The chitosans dissolved in acetate buffer which made up with the following composition (Dawson et al., 1986) [11]: 0.4 $\mathrm{MCH}_{3} \mathrm{COONa} / 0.4 \mathrm{M} \mathrm{CH}_{3} \mathrm{COOH} / 0.2 \mathrm{M}$ $\mathrm{NaCl}$. To keep stable pH 4.5 and ionic strength 0.1 of solution during all experiments the dialyzed with polymer solution the buffer solution was used.

\subsection{Analysis}

\subsubsection{Analytical Ultracentrifugation}

An Optima XL-A analytical ultracentrifuge (Beckman, Palo Alto, CA, USA) was used for all the experiments. It included two integrated detection systems, scanning UV:vis and Rayleigh interference optics systems. For the sedimentation analysis the rotor speed of 50,000 rpm, temperature of $20^{\circ} \mathrm{C}$ and scanning wavelengths of $210 \mathrm{~nm}-270 \mathrm{~nm}$ were employed. Sedimentation coefficient distributions were calculated using of SEDFITBETA2 data evaluation program.

\subsubsection{UV-Spectroscopy}

UV/VIS Spectrometer Lambda 2 (Perkin Elmer) was used for determining of efficiency of the fluorescence labelling reaction of chitosans and to calculate the extinction coefficient of chitosan solutions.

Table 1. Loss on drying (LOD), Degree of acetylation (DA), the solvent density $(\rho)$, partial-specific volume $(v)$ and molecular weight (MW) of used Chitosansamples.

\begin{tabular}{cccccc}
\hline $\begin{array}{c}\text { Chitosan } \\
\text { samples }\end{array}$ & LOD, \% & DA, \% & $\rho(\mathrm{g} / \mathrm{ml})$ & $v(\mathrm{ml} / \mathrm{g})$ & $\mathrm{MW}, \mathrm{g} / \mathrm{mol}$ \\
\hline Chitosan-1 & 9.90 & 85 & 1.817 & 0.550 & 100,000 \\
Chitosan-2 & 7.53 & 82 & 2.000 & 0.465 & 50,000 \\
\hline
\end{tabular}




\subsubsection{Dialyzing and Freeze Drying}

All dialyzing equilibrium of polymer solutions was performed with Spectra Por Dialysis Membrane, MWCO 3500. Vacuum drying of samples was performed with equipment CHRIST LOC-1M, The freezing conditions was performed in vacuum 0.431 mbar and temperature $20^{\circ} \mathrm{C}$.

\subsubsection{The Coupling Reaction}

The modification of chitosan with FITC realized by method described in works Coelfen [10] and Tømmeraasa [12]. It is expected that, coupling of FITC will occur through amination of isothiocyanate by amino group of chitosan [12] [13].

Preliminary dissolved the FITC in DMSO is added to solution of chitosan in acetate buffer $0.4 \mathrm{MCH}_{3} \mathrm{COOH} / 0.4 \mathrm{MCH}_{3} \mathrm{COONa} / 0.2 \mathrm{MNaCl}$. The amount of the reacting compounds correlated as for the first case to 100 structured units of chitosan corresponded one molecule of FITC (DS 1.0\%) and in the second case to 200 units corresponded one molecule of FITC (DS 0.5\%). The reactionary mixture was stirred $24 \mathrm{~h}$. Excesses amount of FITC removed with dialyzing in system Acetic buffer/DMSO taken in 12:1 ratio. With evaporation of the solution under vacuum and low temperature, the sample of fluorescent chitosan is received.

\section{Results and Discussion}

The fluorescein 5-isotiocyanatewas introduced to the different chitosans (Table 1). Theconditions of these reactions kept as it is described in works [11] [14] for similar reaction system. The condensation reaction of a primary amino group (nucleophile) of chitosan (I) is the nucleophile condensing with the carbonyl group of fluorescein 5-isotiocyanate (II), resulting in the secondary amine of the fluorescent chitosan (III) as shown in Scheme 1.

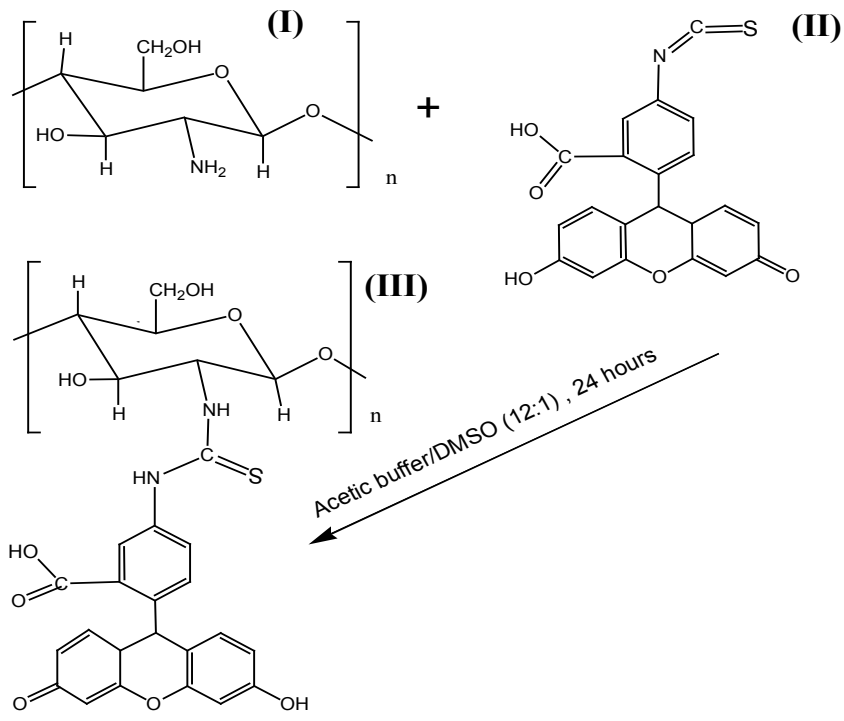

Scheme 1. Reductive amination by chitosan (I) of fluorescein 5-isotiocyanate (II) to obtain the fluorescent chitosan (III). 
The molar ratios of compounds in the coupling reaction as mentioned before were 100:1, 200:1 in order to obtain the chitosans with a chromophore which can conveniently be visualized and quantified by UV or fluorescence spectroscopy, without altering the conformation of the chitosan.

The qualitative estimation of productivity to reactions was performed with UV spectroscopy measurements of modified chitosans solutions with different concentrations in acetate buffer. The maximum emission wavelength of $240 \mathrm{~nm}$ with excitation $\varepsilon=9.934$ was found in specters. Fluorescent chitosans with DS $0.5 \%$ show the same maximum emission wavelength of $240 \mathrm{~nm}$ but with decreased excitation. No significant difference was observed between the maximum emission wavelengths $\left(\varepsilon_{\max }\right)$ of different MW chitosans.

The quantity estimation of degree substitution of the fluoroagent is definite with measuring of compactness charge in macromolecule with the help of devise ParticleCarge Detector PCD 3. According to this measurement for Chitosan-1 with DS $1 \%$ it was $322.556 \mathrm{k} / \mathrm{g}$ and for Chitosan-1 with DS $0.5 \%$ was $162.52 \mathrm{k} / \mathrm{g}$.

The fluorescent chitosans were characterized and some compared analysis of the sedimentation coefficients as a function of the degree of substitution of the chromophore and UV extinction for the two chitosans by treatment of UV absorption scans of the XL-A optic system of the analytical ultracentrifuge was carried out. The data of measurements show that for the chitosan-1 with DA $85 \%$, MW $=100,000$ and substituted with fluoroagent $0.5 \%$ in macrochain has average sedimentation coefficient value $2.3 \mathrm{Svb}$ and extinction coefficient at $240 \mathrm{~nm}$ wavelength of UV corresponded to 5.582. For this chitosan sample with contents of fluoreagent $1 \%$ the sedimentation coefficient is negligibly increased, at extinction coefficient increased on 9.934 as it was expected. For another chitosan sample with DA $82 \%, \mathrm{MW}=50,000$ also observed the same sequence of dependence of the sedimentation coefficient $(\mathrm{S}=1.49 \mathrm{Svb})$ with DS and extinction coefficient.

Sedimentation behavior of fluorescein modified chitosans determines that the chromophor group does not render the essential influence upon molecular and hydrodynamic parameters of chitosan. In the following discussion, we have chosen the modified chitosan-1 (see Table 1) with DS $1 \%$ as an arbitrary example.

As mentioned before, the fluorescein modified chitosan intended to use to obtain the membrane by synthetic boundary method in the analytical ultracentrifuge through interpolymer interactions [8]. In this connection, evaluation of UV absorption properties in different conditions is necessary. Absorption scan exhibit a meniscus peak. This would probably influence the detection of the initial membrane growth at the meniscus interface. Therefore, the occurrence of such a meniscus peak is dependent on the solution concentration range of $1 \%$ $2 \%$ (Figure 1), the wavelength range of $210-270$ (Figure 2) and the run velocity range of $3000-10,000$ (Figure 3) was studied. As an example, for the detection of chitosan for the membrane formation in synthetic boundary method in the 
analytical ultracentrifuge in optimum was assigned to a velocity range 3000 $5000 \mathrm{rpm}$ and wavelength of $240 \mathrm{~nm}$. These scans follow that the obtained fluorescein modified chitosan might be identified and analyzed by UV optics of AUC during membrane formation.

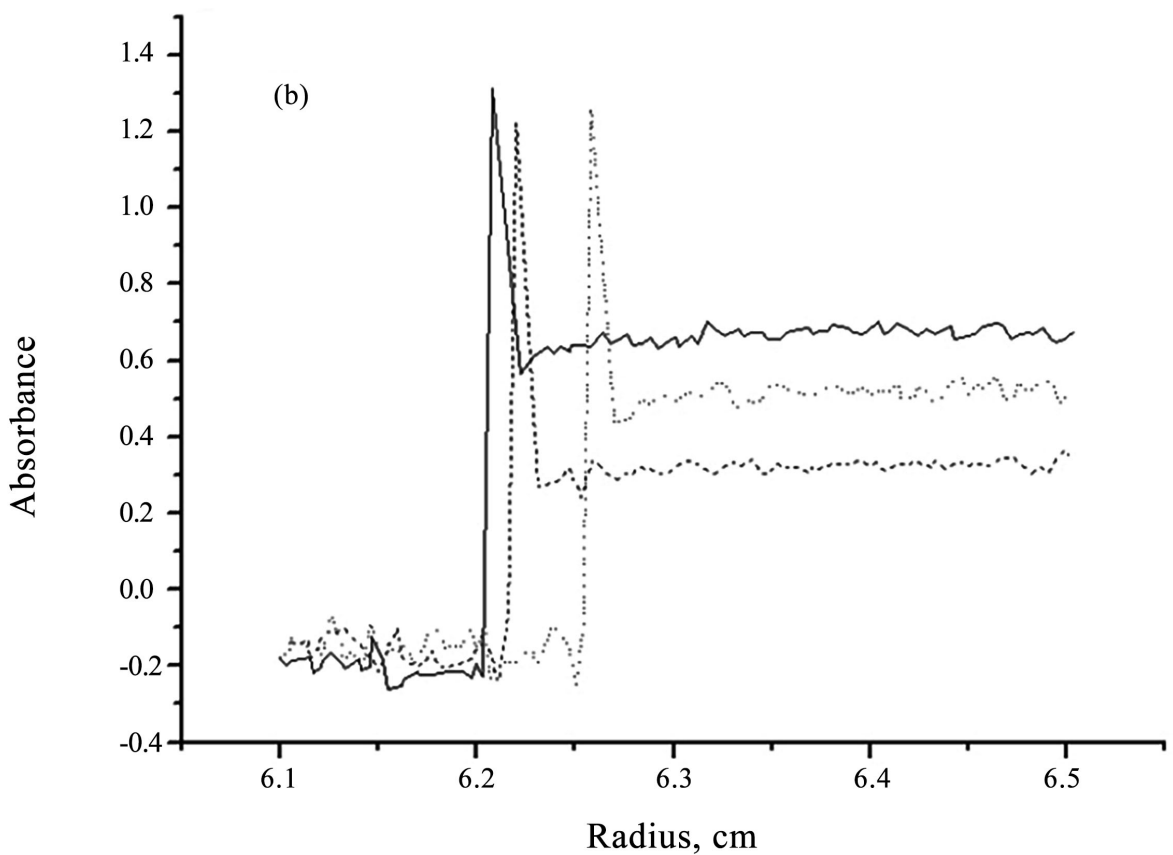

Figure 1. Meniscus detection as function of chitosan concentration: $2.0 \%(-) ; 1.5 \%(\cdots .$.$) ;$ $1.0 \%$ (- - -). The velocity $3000 \mathrm{rpm}$; the wavelength $240 \mathrm{~nm}$.

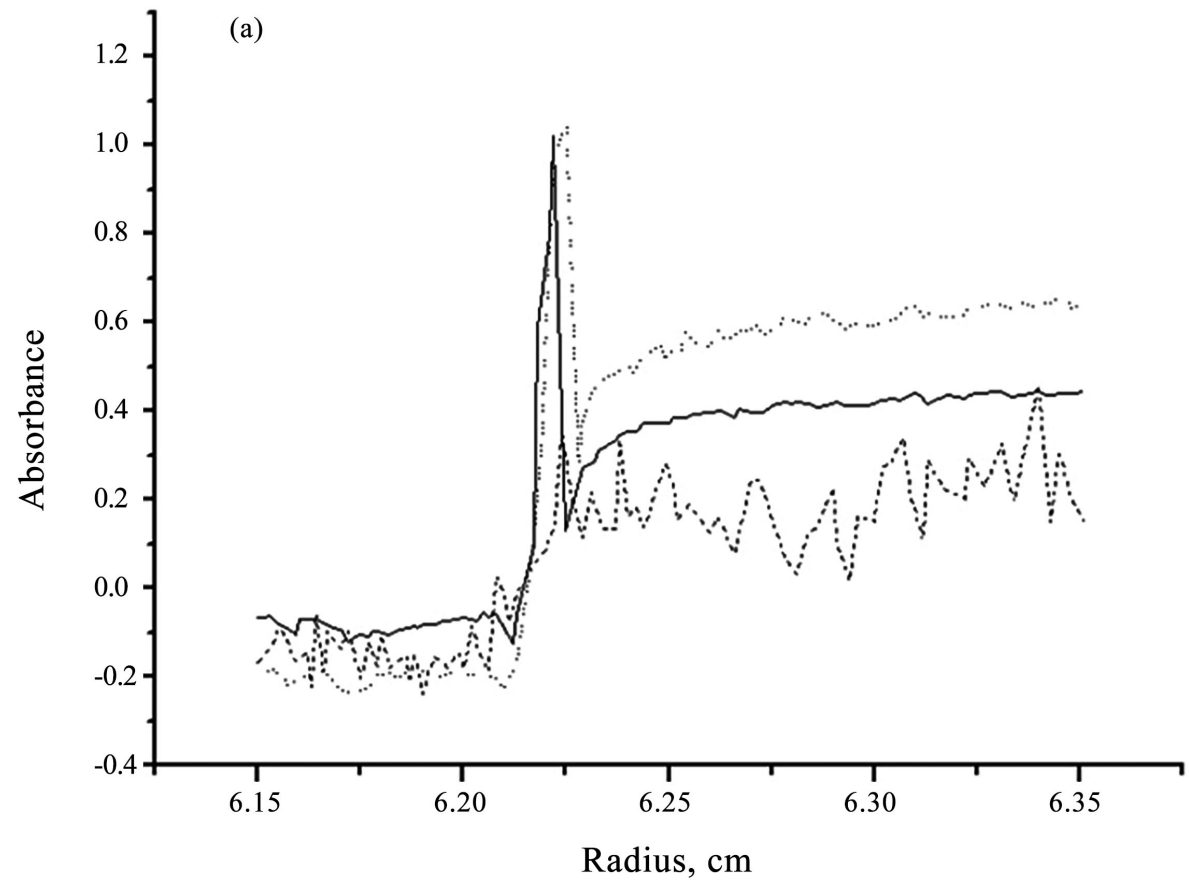

Figure 2. Meniscus detection as function of wavelength: $210 \mathrm{~nm}(-) ; 240 \mathrm{~nm}(\cdots \cdots) ; 270$ $\mathrm{nm}$ (- - -). Chitosan concentration 2\%; the velocity $3000 \mathrm{rpm}$. 


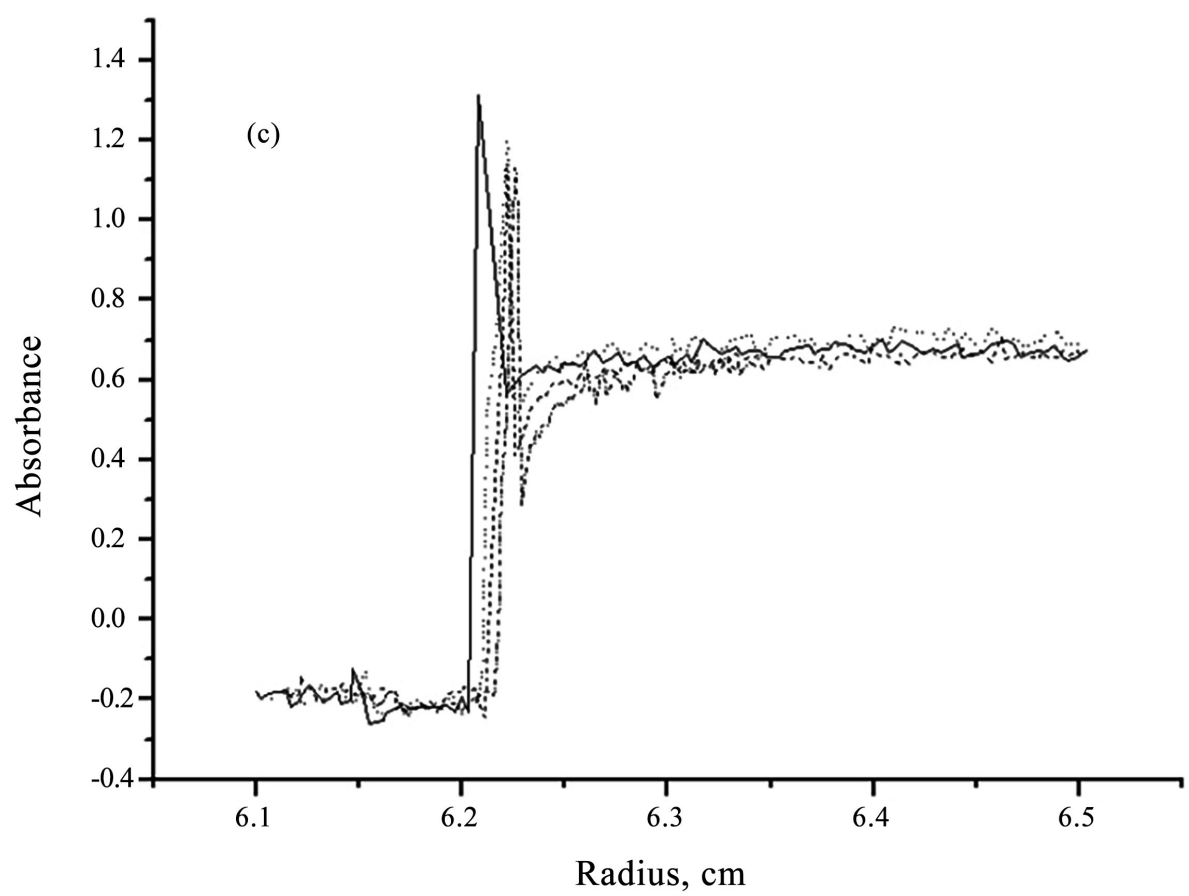

Figure 3. Meniscus detection as function of run velocity: $3000 \mathrm{rpm}(-) ; 5000 \mathrm{rpm}(\cdots .$.$) ;$

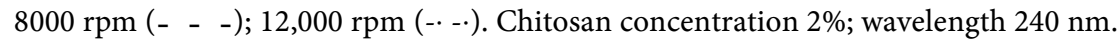

\section{Conclusions}

The fluorescent chitosan without significant depolymerisation of the polysaccharide with high extinction was prepared. Like chitosan, fluorophore 5-isotioceanat chitosan was water soluble at acidic $\mathrm{pH}$ values.

The increased incorporation of the fluorophore 5-isotioceanat at least up to a degree of substitution of $1 \%$ has no deleterious effects on the molar mass of two chitosans of differing degrees of acetylation. The inclusion of the fluorophore allows the use of the absorption optical system of the analytical ultracentrifuge to evaluate the sedimentation velocity and membrane formation process in synthetic boundary methods, for which these samples of chitosan will be used.

\section{Acknowledgements}

I express my gratitude to Professor of University of Konstanz, Germany Helmut Coelfen who provided laboratories and help on carrying out this work, also I thank the professor of Institute of chemistry and physics of polymers of the Academy sciences of Uzbekistan Sayora Rashidova for her assistance.

\section{Conflicts of Interest}

The author declares no conflicts of interest regarding the publication of this paper.

\section{References}

[1] Roberts, G.A.F. (1992) Chitin Chemistry. Macmillan Press Ltd., Hong Kong, 368 p. 
https://doi.org/10.1007/978-1-349-11545-7

[2] Kean, T., Roth, S. and Thanou, M. (2005) Trimethylated Chitosans as Non-Viral Gene Delivery Vectors: Cytotoxicity and Transfection Efficiency. Journal of Controlled Release, 103, 643-653. https://doi.org/10.1016/j.jconrel.2005.01.001

[3] Roberts, G.A.F. (2008) Thirty Years of Progress in Chitin and Chitosan. In: Jaworska, M.M., Ed., Progress on Chemistry and Application of Chitin and Its Derivatives, Polish Chitin Soc., Lodz, Vol. 13, 7-15.

[4] USDA NOP and EPA Rule on Chitosan (2007) Federal Register No. 236, Vol. 72.

[5] Chitin and Chitosan Final Registration Review Decision. Document ID: EPA-HQ-OPP-2007-0566-0019, 10-15.

[6] Rashidova, S.Sh. and Milusheva, R.Yu. (2009) Chitin and Chitosan of Bombyx Mori: Synthesis, Properties and Applications. Fan, Tashkent, 246 p. (In Russian)

[7] Wandrey, C. and Bartkowiak, A. (2001) Membrane Formation at Interfaces Examined by Analytical Ultracentrifugation Techniques. Colloids and Surfaces A: Physicochemical and Engineering Aspects, 180, 141-153. https://doi.org/10.1016/S0927-7757(00)00767-6

[8] Bourdillon, L. and Wandrey, C. (2004) On-Line Study of Polyelectrolyte Network Formation by Interfacial Reaction. Colloid and Polymer Science, 282, 1247-1257. https://doi.org/10.1007/s00396-004-1076-5

[9] Cunha-Reis, C., El Haj, A.J., Yang, X.B. and Yang, Y. (2013) Fluorescent Labeling of Chitosan for Use in Non-Invasive Monitoring of Degradation in Tissue Engineering. Tissue Engineering and Regenerative Medicine, 7, 39-50. https://doi.org/10.1002/term.494

[10] Coelfen, H., Harding, S.E. and Varum, K.-M. (1996) Investigation, Using Analytical Ultracentrifugation, of the Effect of the Incorporation of the Fluorophore 9-Anthraldehyde on Two Chitosans of Differing Degrees of Acetylation. Carbohydrate Polymers, 30, 55-60. https://doi.org/10.1016/S0144-8617(96)00042-2

[11] Dawson, R.M.C., Elliott, D.C., Elliott, W.H. and Jones, K.M. (1986) Data for Biochemical Research. 3rd Edition, Oxford University Press, Oxford, 429 p.

[12] Tømmeraasa, K., Stranda, S.P., Tianb, W., Kenneband, L. and Vårum, K.M. (2001) Preparation and Characterisation of Fluorescent Chitosans Using 9-Anthraldehyde as Fluorophore. Carbohydrate Research, 336, 291-296.

[13] Yalpani, M. and Hall, L.D. (1981) Synthesis of Fluorescent Probe-Carbohydrate Conjugates. Canadian Journal of Chemistry, 59, 2934-2939. https://doi.org/10.1139/v81-425

[14] Borch, R.F., Bernstein, M.D. and Dupont Durst, H. (1971) Cyanohydridoborate Anion as a Selective Reducing Agent. Journal of the American Chemical Society, 93, 2897-2904. https://doi.org/10.1021/ja00741a013 